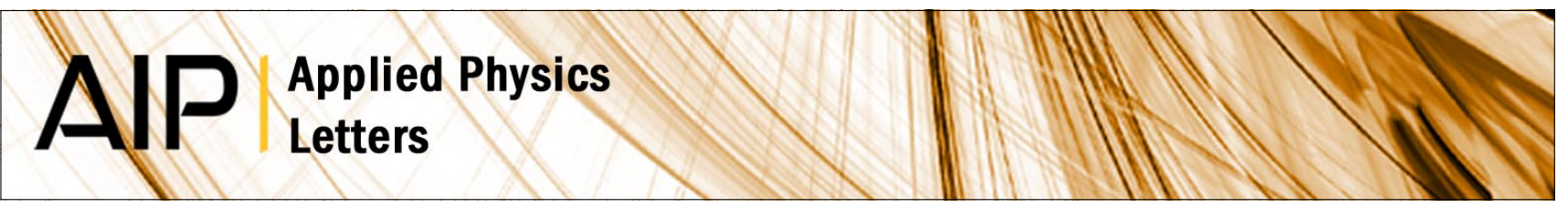

\title{
Femtosecond-irradiation-induced refractive-index changes and channel waveguiding in bulk Ti3+:Sapphire
}

V. Apostolopoulos, L. Laversenne, T. Colomb, C. Depeursinge, R. P. Salathé et al.

Citation: Appl. Phys. Lett. 85, 1122 (2004); doi: 10.1063/1.1781737

View online: http://dx.doi.org/10.1063/1.1781737

View Table of Contents: http://apl.aip.org/resource/1/APPLAB/v85/i7

Published by the American Institute of Physics.

\section{Related Articles}

Pump dependence of the dynamics of quantum dot based waveguide absorbers Appl. Phys. Lett. 100, 241108 (2012)

Experimental demonstration of ultra-compact directional couplers based on silicon hybrid plasmonic waveguides Appl. Phys. Lett. 100, 241105 (2012)

Realizing almost perfect bending waveguides with anisotropic epsilon-near-zero metamaterials Appl. Phys. Lett. 100, 221903 (2012)

Photonic crystal tunable slow light device integrated with multi-heaters Appl. Phys. Lett. 100, 221110 (2012)

Control of tensile strain in germanium waveguides through silicon nitride layers Appl. Phys. Lett. 100, 201104 (2012)

\section{Additional information on Appl. Phys. Lett.}

Journal Homepage: http://apl.aip.org/

Journal Information: http://apl.aip.org/about/about_the_journal

Top downloads: http://apl.aip.org/features/most_downloaded

Information for Authors: http://apl.aip.org/authors

\section{ADVERTISEMENT}

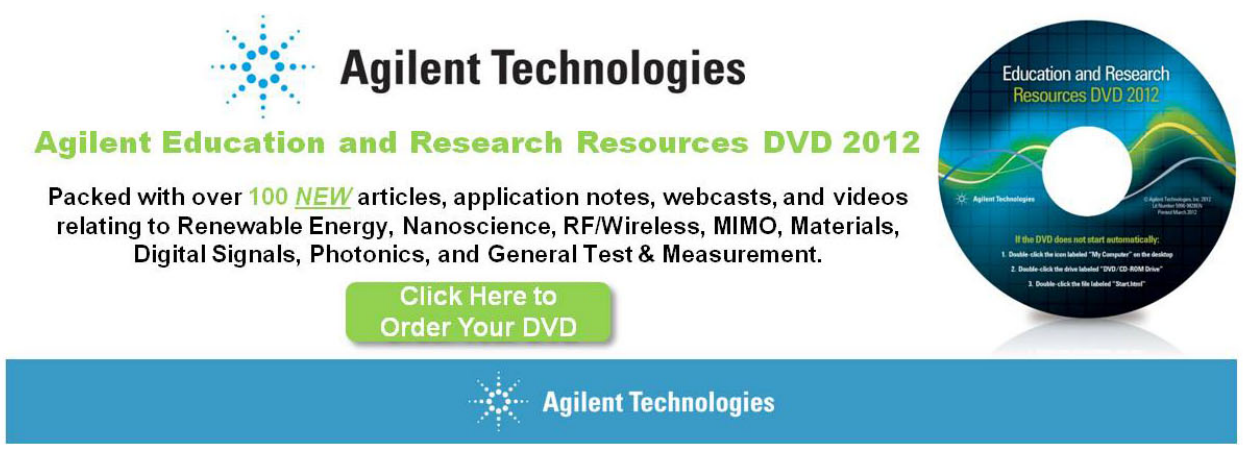




\title{
Femtosecond-irradiation-induced refractive-index changes and channel waveguiding in bulk $\mathrm{Ti}^{3+}$ :Sapphire
}

\author{
V. Apostolopoulos, ${ }^{\text {a) }}$ L. Laversenne, T. Colomb, C. Depeursinge, R. P. Salathé, and \\ M. Pollnau \\ Advanced Photonics Laboratory, Institute for Imaging and Applied Optics, \\ Swiss Federal Institute of Technology, CH-1015 Lausanne, Switzerland \\ R. Osellame, G. Cerullo, and P. Laporta \\ Dipartimento di Fisica, Politecnico di Milano and IFN-CNR, Piazza Leonardo da Vinci 32, \\ I-20133 Milano, Italy
}

(Received 5 January 2004; accepted 15 June 2004)

\begin{abstract}
We have employed femtosecond laser writing in order to induce refractive-index changes and waveguides in $\mathrm{Ti}^{3+}$-doped sapphire. Doping the sapphire crystal with an appropriate ion significantly reduces the threshold for creating structural changes, thus enabling the writing of waveguide structures. Passive and active buried channel waveguiding is demonstrated and images of the guided modes, propagation-loss values, fluorescence spectra, and output efficiencies are presented. The guiding area is located around the laser-damaged region, indicating that the guiding effect is stress induced. Refractive-index changes are measured by digital holography. Proper active doping should enable femtosecond processing and waveguide writing in various crystalline materials. (C) 2004 American Institute of Physics. [DOI: 10.1063/1.1781737]
\end{abstract}

Nonlinear absorption of femtosecond laser pulses has been employed in order to induce structural changes by microexplosions in numerous materials ${ }^{1}$ and also for fabricating waveguide structures. ${ }^{2-4}$ Femtosecond pulses can be used for precise micromachining of materials, as the pulse energy is transferred to electrons and the optical excitation ends before the lattice is perturbed. The energy deposition is based on multiphoton absorption and avalanche ionization; the nonlinearity of the process can be exploited in order to write structures into the bulk of materials. Therefore, femtosecond writing provides the possibility of implementing threedimensional (3D) optical integrated circuits. Another advantage of this fabrication process is the capability of rapid prototyping of a device without the need for any photolithographic process.

Waveguides in $\mathrm{Ti}^{3+}$ : Sapphire are of great importance for applications as low-threshold tunable lasers, integrated femtosecond lasers, and as broadband light sources in optical coherence tomography. ${ }^{5}$ Surface channel waveguides in hard crystalline materials, such as sapphire and $\mathrm{Ti}^{3+}:$ Sapphire, have been obtained by a number of methods that require the use of photolithographic masks, such as reactive ion etching ${ }^{6}$ of planar waveguides, ion indiffusion, ${ }^{7}$ as well as in-depth channel waveguides by proton implantation. ${ }^{8}$ Here, we report writing of waveguides by femtosecond pulses in a hard crystalline material, $\mathrm{Ti}^{3+}:$ Sapphire. Waveguiding is observed around microdamaged areas induced by femtosecond irradiation. We found that the threshold for creating microdamage by femtosecond irradiation in sapphire is greatly decreased when the crystal is doped with $\mathrm{Ti}^{3+}$ ions. The process also shows promise for 3D integrated circuits in other hard crystalline materials when sensitized by an appropriate doping ion.

Two writing systems using $\mathrm{Ti}^{3+}$ : Sapphire lasers at repetition rates of $1 \mathrm{kHz}$ and $25 \mathrm{MHz}$, respectively, with a cen-

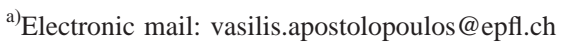

ter pulse wavelength of $790 \mathrm{~nm}$, were employed. For the low-repetition-rate system, the pulse duration was $150 \mathrm{fs}$ and the pulse energy was varied from 0.5 to $6 \mu \mathrm{J}$, whereas the maximum pulse energy available from the high-repetitionrate system was $30 \mathrm{~nJ}$ with pulse duration $<30$ fs. For focusing the laser beam, a 0.3 numerical aperture (NA) microscope objective was used with the $\mathrm{kHz}$ system; for the $\mathrm{MHz}$ system, a 1.4 NA oil-immersion objective was employed. The focal point was inside the bulk of the material (100 to $300 \mu \mathrm{m}$ deep) with a spot size of approximately $10 \mu \mathrm{m}$ (0.3 NA objective) and $\sim 1 \mu \mathrm{m}$ (1.4 NA objective). The sample was held on a translation stage that moved in a direction perpendicular to the writing beam; the writing speed was varied from 9 to $17 \mu \mathrm{m} / \mathrm{s}$ (kHz system) and from 0.1 to $10 \mathrm{~mm} / \mathrm{s}$ (MHz system). One sample of pure $c$-cut sapphire and one sample of $\mathrm{Ti}^{3+}$ :Sapphire with a $\mathrm{Ti}^{3+}$ concentration of 0.21 at. \% were irradiated. When employing the high-repetition-rate system, none of the irradiated samples showed any effect. With the higher energies available from the low-repetition-rate system, the undoped sapphire did not exhibit any damage or refractive-index modification, whereas channels approximately $10 \mathrm{~mm}$ in length were written inside the bulk of the $\mathrm{Ti}^{3+}$ : Sapphire sample.

In Fig. 1(a), a microscope image of the end face of two irradiated regions in the $\mathrm{Ti}^{3+}$ : Sapphire sample is shown. Using the technique of digital holography, ${ }^{9,10}$ the optical path differences in a 1.1-mm thick slice cut from the irradiated sample were monitored with the probing laser beam passing through the sample in the waveguide direction. Two line profiles were taken, line (I) slightly above and line (II) directly through the damaged regions, as indicated in Fig. 1(a). From the measured optical path differences, see Fig. 1(b), positive and negative refractive-index changes of approximately 1 and $-2 \times 10^{-4}$ were obtained in the regions above the tips of the damaged regions and directly in the damaged regions, respectively. 

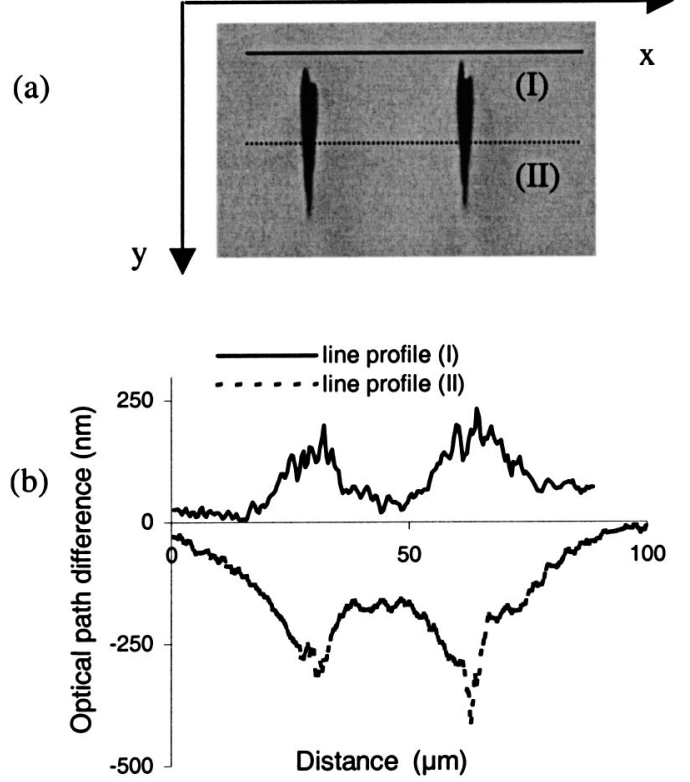

FIG. 1. (a) Microscope image of the end face of the irradiated $\mathrm{Ti}^{3+}$ : Sapphire sample. (b) Optical path difference measurements using the technique of digital holography, performed in the waveguide direction while scanning along the lines (I) and (II) indicated in (a).

Consequently, the waveguides were always formed in those regions of the $\mathrm{Ti}^{3+}$ :Sapphire sample that are adjacent to the upper and lower tips of the regions damaged by the femtosecond irradiation. The light of a $\mathrm{He}-\mathrm{Ne}$ laser at $633 \mathrm{~nm}$ was coupled into the polished end face of the sample using a microscope objective. Two mode images of guided light at $633 \mathrm{~nm}$ in transverse electric (TE) polarization recorded by a charge coupled device (CCD) camera are shown in Fig. 2. TE and transverse magnetic polarizations possessed slightly different mode distributions, however the polarization dependence was not significant. Also, an Ar-ion laser operating on all visible lines was launched into the waveguide in $\pi$-polarization in order to pump the active $\mathrm{Ti}^{3+}$ ions. The guided infrared fluorescence at the output of the waveguide was measured using an optical spectrum analyzer. The spectral shapes of fluorescence emitted from waveguide and bulk regions of the $\mathrm{Ti}^{3+}$ :Sapphire sample are identical, see Fig. 3(a), indicating that the $\mathrm{Ti}^{3+}$ fluorescence is not quenched by irradiation-induced strain. From the measured fluorescence output power of the waveguide versus pump power of the Ar-ion laser coupled into the waveguide with a $6.3 \times$ microscope objective, see Fig. 3(b), we calculated a guided fluorescence efficiency of $3.4 \times 10^{-5}$, which is similar to the value of $4.1 \times 10^{-5}$ reported in reactive ion etched $\mathrm{Ti}^{3+}$ :Sapphire surface channel waveguides. ${ }^{6}$

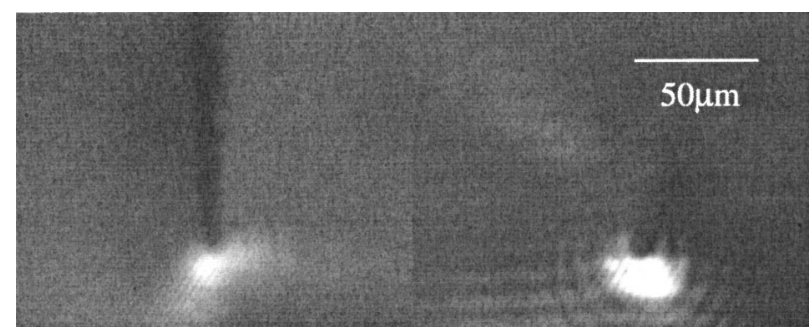

FIG. 2. Mode profiles $(633 \mathrm{~nm}$, TE polarization) of two different waveguides written into the bulk of the $\mathrm{Ti}^{3+}$ :Sapphire sample. (Visible diagonal fringes are due to a detection artifact.) (a)

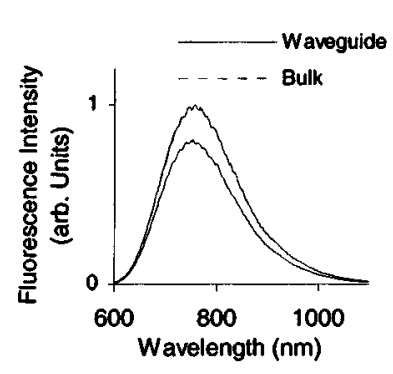

(b)

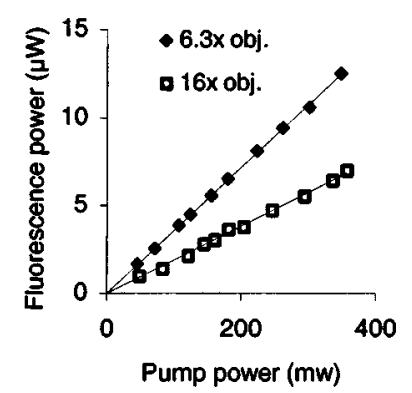

FIG. 3. Fluorescence output collected from a waveguide written into the bulk of the $\mathrm{Ti}^{3+}$ :Sapphire sample: (a) Comparison of spectral fluorescence shapes from waveguide and bulk regions; and (b) input-output curves obtained by coupling the pump with two different microscope objectives.

The propagation loss of the waveguide was measured by exciting the $\mathrm{Ti}^{3+}$ ions with guided Ar-ion laser light, recording the intensity of the streak of fluorescence emitted perpendicular to the waveguide with a CCD camera, fitting an exponential loss curve, and subtracting the absorption loss of the Ar-ion laser light measured in the bulk of the sample. Alternatively, $\mathrm{He}-\mathrm{Ne}$ laser light at $633 \mathrm{~nm}$ was coupled through a single-mode fiber into the waveguide. The same single-mode fiber and a multimode fiber were used to collect the output of the waveguide. Assuming that the multimode fiber has negligible coupling loss, the coupling loss of light from the single-mode fiber to the waveguide can be estimated; thus, the propagation loss of the waveguide was found. The loss values obtained with these two methods are 2.3 and $2.5 \mathrm{~dB} / \mathrm{cm}$, respectively. The second method gives accurate results only for single-mode waveguides; in our case, where the waveguide is multimode at $633 \mathrm{~nm}$, it is used to confirm the value obtained with the fluorescence streak measurement.

The fact that femtosecond irradiation using the same parameters did not show any effect in undoped sapphire indicates that the threshold for creating microdamage by femtosecond irradiation is greatly decreased when sapphire is doped with $\mathrm{Ti}^{3+}$ ions. The most probable reason is that a double two-photon absorption, first, into the dopant's absorption band in the blue-green spectral region and, second, from there into the band gap of the host, has a higher probability than four-photon absorption directly into the band gap of the host. In addition, the lattice distortion initially introduced to the sapphire lattice by the $\mathrm{Ti}^{3+}$ dopant, which is larger than the substituted $\mathrm{Al}^{3+}$ ion, may support the mechanism that induces the observed microdamage. This recipe of sensitizing the host material by an optically active ion may well be employed also for other hard crystalline materials.

In glasses, waveguiding occurs due to melting and fast resolidification resulting in higher density, thus providing waveguiding in the exposed region. In contrast, the damage induced in sapphire decreases the lattice order and creates an amorphous or polycrystalline region in the irradiated area, resulting in the measured decrease of the refractive index, see Fig. 1(b), line (II). Therefore, no waveguiding can occur in the damaged region. However, since the lower density of the amorphous compared to the crystalline phase results from an expansion of the damaged region, stress occurs at the boundaries between the damaged and the nondamaged regions, thus creating a refractive-index increase close to the 
damaged region, see Fig. 1(b), line (I), and consequently waveguiding. ${ }^{4}$

Femtosecond laser writing was also performed using astigmatic beam focusing optics in order to create symmetrical circularly damaged areas; ${ }^{3}$ nevertheless, the best light confinement was observed near the tips of asymmetrically damaged areas (Fig. 2). The reason is to be attributed to a more concentrated stress near sharp angles that allows localized waveguiding, while a more distributed index increase is not able to provide good confinement.

In conclusion, active waveguides directly written by femtosecond irradiation have been demonstrated in a $\mathrm{Ti}^{3+}$ :Sapphire crystal. It has been shown that refractiveindex modifications and channel waveguides can be written into the bulk of a hard crystalline material by sensitizing the crystal with an appropriate dopant ion. Future work will concentrate on investigating the dependence of the waveguide characteristics on fabrication parameters, such as pulse energy and focusing optics. A systematic study will lead to buried waveguides with controlled modal characteristics for the implementation of an integrated waveguide laser or a two-dimensional array of active waveguides to be used as a light source for parallel optical coherence tomography. ${ }^{11}$
Studying the physical origin of the process will also give useful information for employing the process to other appropriately doped crystalline materials for fabrication of 3D optical circuits.

${ }^{1}$ E. N. Glezer and E. Mazur, Appl. Phys. Lett. 71, 882 (1997).

${ }^{2}$ K. M. Davies, K. Miura,N. Sugimoto, and K. Hirao, Opt. Lett. 21, 1729 (1996).

${ }^{3}$ R. Osellame, S. Taccheo, M. Marangoni, R. Ramponi, P. Laporta, D. Polli, S. De Silvestri, and G. Cerullo, J. Opt. Soc. Am. B 20, 1559 (2003).

${ }^{4}$ T. Gorelik, M. Will, S. Nolte, A. Tünnermann, and U. Glatzel, Appl. Phys. A: Mater. Sci. Process. 76, 309 (2003).

${ }^{5}$ M. Pollnau, J. Lumin. 102, 797 (2003).

${ }^{6}$ A. Crunteanu, M. Pollnau, G. Jänchen, C. Hibert, P. Hoffmann, R. P. Salathé, R. W. Eason, C. Grivas, and D. P. Shepherd, Appl. Phys. B: Lasers Opt. 75, 15 (2002).

${ }^{7}$ V. Apostolopoulos, L. M. B. Hickey, D. A. Sager, and J. S. Wilkinson, Opt. Lett. 26, 1586 (2001).

${ }^{8}$ L. Laversenne, P. Hoffmann, M. Pollnau, and P. Moretti, Conference on Lasers and Electro-Optics, Technical Digest (Optical Society of America, Washington, D.C., 2004), paper CTuU5.

${ }^{9}$ E. Cuche, F. Bevilacqua, and C. Depeursinge, Opt. Lett. 24, 291 (1999).

${ }^{10}$ E. Cuche, P. Marquet, and C. Depeursinge, Appl. Opt. 38, 6994 (1999).

${ }^{11}$ M. Ducros, M. Laubscher, B. Karamata, S. Bourquin, T. Lasser, and R. P. Salathé, Opt. Commun. 202, 29 (2002). 